\title{
The quantitative and molecular genetics of individual differences in animal personality
}

Veronika N Laine and Kees van Oers

Department of Animal Ecology, Netherlands Institute of Ecology (NIOO-KNAW), P.O. Box 50, 6700 AB, Wageningen, The Netherlands

\begin{abstract}
:
One of the main goals in current personality research is to identify genes behind the measured behavioural variations. This is important in order to study how under the influence of the environment, gene expression changes are translated into the observed phenotypes. The advances, especially in genomic technologies, have made it possible to identify genetic loci behind these variations, also concerning nonmodel species. In this chapter, we will describe the role and relevance of quantitative and molecular genetic approaches in explaining the existence and maintenance of variation in animal personality. We here will provide 1) a timely review on the papers published on this topic, 2) an overview of the current situation and progress, and 3) a view on the likely new avenues the field will take.
\end{abstract}

Keywords: personality, genetics, behaviour, QTL mapping, genome-wide association studies, pleiotropy, quantitative genetics, molecular genetics, polygenic trait 


\section{Introduction}

It has now been established that, just like in humans, individuals of a wide range of animal species express variation in personality. Animal personality describes between-individual differences in behaviours that are consistent over context and time (Carere \& Maestripieri, 2013). Often these personality traits correlate and thereby form suites of traits, so-called behavioural syndromes (Sih et al., 2004). Whereas the phenomenon of individual consistency in behavioural traits was described before (e.g., Burtt, 1973; Williams et al., 1962), there has been a large increase in papers that attempt to explain the causes and consequences of variation in personality traits over the past three decades (see for overview: Carere \& Maestripieri, 2013). The study of animal personality has now grown to be a multidisciplinary field, which unites scientists who use various approaches to study animal behaviour and work in fields ranging from comparative psychology to neuroscience and evolutionary biology.

When trying to explain the origin of variation in personality traits an immediate question is to what extent variation in personality traits can be explained by genes, the environment, and the interaction between the two. Where genetic studies will provide information on the genomic structure and mechanisms, possible constraints, and the potential for natural selection to act on different levels of variation (van Oers \& Sinn, 2013), such information is valid only when tested along axes of relevant environmental variation. Already from early studies, it was obvious that both genes and the environment play important roles in describing variation in behaviours, but their relative contribution and importance has been a point of discussion since then 
(see e.g., Groothuis \& Trillmich, 2011; van Oers et al., 2005). In more recent years this discussion has changed into a more interactive one, in which it is recognized that the absolute role for genes and environment cannot be separated and the integration rather than the contrast between the two will yield new insights (Groothuis \& Trillmich, 2011; van Oers \& Sinn, 2011).

In this chapter, we will describe the role and relevance of quantitative and molecular genetic approaches in explaining the existence and maintenance of variation in animal personality. Although several reviews and some book chapters have been written on this topic (Dochtermann \& Roff, 2010; Dochtermann et al., 2015; van Oers et al., 2005, van Oers \& Mueller, 2010; van Oers \& Sinn 2011, 2013), we here will provide 1) a timely review on the papers published on this topic, 2) an overview of the current situation and progress, and 3) the likely new avenues the field will take.

\section{Theory of genetic influence of animal personality}

The identification of a heritable factor is the essential starting point for all evolutionary research on any behavioural trait (Boake et al., 2002). Part of the phenotypic variation that is observed in behavioural traits in animals is transmitted across generations (Stirling et al., 2002). The presence of some level of heritability, where resemblance can be observed among relatives across or within generations, has been identified in many studies, regardless of the mechanism that may be responsible for this resemblance. Traditionally, "heritable variation", "genetic variation", and "genomic variation" were envisioned as interchangeable terms. Genetic variation due to genomic polymorphisms was viewed as the basis for individual differences in the expression of behaviours, and phenotypic plasticity, i.e., 
the residual irreversible and reversible phenotypic variance, was assigned to the interaction of the expression of the genes with the environment, due to, for example, development and learning (Arnold et al., 2007; Brydges et al., 2008; Quinn \& Cresswell, 2005). Selection was thereby expected to be acting only on the additive genetic component, reflected in the so-called narrow-sense heritability estimate. This is in contrast to the broad-sense heritability, which, apart from the additive genetic component, includes other components that may contribute to resemblance among relatives, like maternal effects, non-additive effects, or epistasis, the interaction between genes (Lynch \& Walsh, 1998). Recent ideas build on the observation that transgenerational inheritance may be caused by processes other than genetic polymorphisms (Danchin et al., 2011) and this view has consequences for how we expect measures of heritability and genomic differences among individuals to be associated with one another. In this chapter we therefore make a clear distinction between quantitative genetics, which may not explicitly take into account the genomic and epigenetic mechanisms responsible for the heritable component, and molecular genetics, in which an intrinsic interest exists in explaining direct causes for changes in gene expression on the genomic level (see below).

\section{Personality as a polygenic trait}

As with many other quantitative traits, many loci with small effects are expected to be involved in animal personality variation. Although this theory is accepted by the majority of researchers, hardly any direct proof for it exists. To our knowledge, only one study specifically tested the polygenetic nature of one personality trait in a wild species (Santure et al. 2015) by combining a chromosome partitioning approach 
(Robinson et al. 2013) and a calculation of the number of loci contributing to the variation (Guan \& Stephens 2011). Both a positive association between the amount of variation explained and the size of a chromosome, and an estimation that hundreds of loci were identified that contributed to the variation in exploratory behaviour, point to the fact that indeed such traits are influenced by many genes of small effect distributed throughout the genome (Santure et al. 2015). These loci are expected to play roles at different organisational levels. There will be sets of genes that will affect behavioural trait variation per se. Polymorphisms in such genes are likely to affect the amount or structure of proteins influencing behaviour in a direct way. Whereas, on the other hand, there will be genes involved in the plasticity a trait expresses (Dingemanse \& Wolf 2013). A more direct cause for the quantitative nature of behavioural traits is the fact that some loci will be involved in the pathway, but will not have genomic variation among individuals (Visser et al. 2010). Such genes may for example be genes that are essential in the pathway, but may only be switched on or off. These genes can therefore not account for genomic variation among individuals, i.e., will not bear any polymorphisms, and thus cannot be identified using conventional molecular genetic approaches (e.g., Zou \& Zeng 2009). However, they can be identified using expression studies, because the expression levels of these genes may be important drivers of a plastic expression of a trait (Brem \& Kruglyak 2005).

\section{Non-additive genetic effects}

Where additive genetic effects describe the independent effects of loci on the trait, in cases when genes interact, non-additive effects may affect the expression and 
inheritance of such traits. Substantial non-additive genetic effects may point to the stabilizing nature of selection acting on personality traits (Penke et al. 2007). Studies have shown that non-additive genetic variance may contribute to substantial amounts of the heritability of behavioural traits in general (Meffert \& Hicks 2002) and personality traits specifically (Adams et al., 2012; Jordan et al., 2007; van Oers et al., 2004), indicating that this might be a general feature of personality traits, explaining some of the variation in heritability estimates (van Oers \& Sinn, 2013). Where the presence of such non-additive genetic effects can be incorporated in quantitative genetic models (see below), studies that test for these effects are still rare. The exact origin of non-additive effects is often difficult to assess and simulations have shown that complex patterns of, for example, epistasis may apparently be there, while the effect of the genes is purely additive (Eaves \& Verhulst, 2014).

\section{Distinction between quantitative and molecular genetics}

Here we want to make a distinction between two approaches that can be used to study heritable variation in traits, including behavioural traits. On the one hand, studies use information on resemblance among individuals to study inheritance patterns of behavioural traits across generations (quantitative genetics). On the other hand, studies can investigate the architecture and function of genomic structures such as genes at a molecular level (molecular genetics). This distinction in methods is important for identifying the level of questions one wants to answer and the implications of the results from these studies. 


\section{Quantitative genetics of animal personality}

The goal in quantitative genetic studies is to identify the fraction of variation in a phenotype that is attributable to a heritable component. Quantitative traits, such as the majority of behavioural traits, are characterized by a continuous distribution, likely caused by polymorphisms in many loci, the expressions of which, and therefore the phenotypes, are responsive to environmental fluctuations.

Genetic studies in captivity often rely on artificial selection of specific traits (e.g., Drent et al., 2003; van Oortmerssen \& Bakker, 1981; van Oers et al., 2004) or crosses between breeds (Laine et al., 2013; van Oers et al., 2013). The so-called narrow-sense heritability derived from such experiments may indicate the evolutionary potential of the traits at a specific time point, given the additive genetic variation of the parental population. While these studies have given us much insight into the genetic mechanisms and the standing genetic variation in certain populations at certain time points, validation and experimental studies are needed in natural populations to assess how these findings relate to variation under natural conditions.

A quantitative genetic analysis in a natural population requires that individuals are measured for their behaviour and that information on the relatedness among those individuals is available (Falconer \& Mackay, 1996, Lynch \& Walsh, 1998, Kruuk et al., 2008). This information is then used to construct statistical models that integrate phenotypic and relatedness information, so-called animal models. Animal models use pedigrees to estimate genetic parameters (Kruuk 2004), such as the additive 
genetic variance $\left(V_{A}\right)$ and the heritability $\left(h^{2}\right)$, the latter being the ratio of $V_{A}$ to the total phenotypic variance (VP). Animal models were originally designed to determine the genetic merit of individuals by calculating the genetic variance components of mainly farm animals for animal breeders (Henderson, 1984) to emphasize specific traits, but were later adopted by quantitative geneticists working on pedigreed natural populations (Kruuk, 2004; Lynch \& Walsh, 1998), and are now also used in personality studies in captive (Careau et al., 2011; van Oers et al., 2004) and natural populations (Brommer \& Kluen, 2012; Dingemanse \& Dochtermann, 2013; Quinn et al., 2009; Taylor et al., 2012).

Quantitative genetic studies in natural populations typically find that between 20 and 50 percent of the phenotypic variation in animal personality traits can be attributable to a heritable component (for reviews see ;Dochtermann et al., 2014; Van Oers et al., 2005; Van Oers \& Sinn, 2013). In a recent example, Petelle et al. (2015) estimated the additive genetic variance and covariance of four personality traits, docility, sociability, activity and exploratory behaviour, in a wild population of yellowbellied marmots (Marmota flaviventris). By using behavioural information from individuals that were part of marker-based pedigree spanning 11 years, they found that about $10-15$ percent of the total phenotypic variation could be explained by an additive genetic component and around 40 percent of the between-individual variance could be explained by resemblance between relatives. However, in a recent meta-analysis including 70 measures of both heritability and repeatability in 10 study systems, Dochtermann et al. (2014) tested the degree to which the repeatable part of behavioural variation could be attributed to additive genetic variation. They found 
that approximately $52 \%$ of the consistent part of animal personality variation was the result of additive genetic variation. This meta-analysis shows that the heritability of personality is higher than previously estimated, which might demonstrate that heritable differences are likely to be a major contributor to variation in animal personality as well as support the phenotypic gambit: that evolutionary inferences drawn from repeatability estimates may often be justified. Furthermore, while additive genetic variation is a primary contributor to personality, considerable variation remains to be explained. The animal model approach also allows for other components of the phenotypic variance to be modelled, such as common environment or maternal effects, often requiring structured experiments and/or multiple measures of the same or related individuals. Brommer and Kluen (2012), for example, conducted a 3-year study of reciprocal cross-fostering within a pedigreed blue tit (Cyanistes caeruleus) population. Heritability estimates of handling aggression, breathing rate, and docility confirmed those found in non-experimental studies ( $h^{2}=16-29 \%$ ), but they were strongly affected by a "nest-of-rearing" component, indicating that the phenotypic similarity of relatives may also be due to common environment effects.

More recently, quantitative genetic methods have been applied to assess more than only the heritable component in personality traits. Two important components of personality research are 1) the multivariate nature of personality traits and 2) the assumption that individuals are consistent over time and context. To integrate these aspects into animal models, both genetic correlations (Dochtermann \& Roff, 2010) 
and the genetic basis of phenotypic plasticity (Dingemanse et al., 2009) can be integrated into these animal models (Adams et al., 2012).

\section{Animal personality trait correlations}

Where individual consistency is an important feature of animal personality, the observed phenotypic correlations often referred to as behavioural syndrome (Sih et al., 2004) is another aspect. Where the genetic structure underlying a trait will provide information on the evolvability of a single trait, traits are not expected to be expressed independently. Nevertheless, estimations of genetic correlations are still rare (but see Dochtermann \& Roff, 2010; Roff, 1996; van Oers et al., 2005). This lack of estimates of genetic correlations calls for the need to view and analyse traits in a multivariate way. Quantitative genetic methods allow for the characterization of this so-called multivariate behavioural phenotype by estimating within, among and independent structures (Dochtermann \& Roff, 2010). In Brommer and Kluen's (2012) study on blue tits, for example, it was specifically tested whether phenotypic correlations provided a correct description of the genetic correlations. They found that the phenotypic and genetic correlations indeed went in the same direction and were roughly of the same size when looking within cohorts. In a follow-up study, however, they concluded that this genetic correlation could disappear during development due to age-specific genetic effects (Class \& Brommer, 2015). Moreover, in yellow-bellied marmots (Marmota flaviventris), researchers found that only one of the phenotypic correlations between the traits could be explained by a positive genetic correlation, indicating that even when traits are correlated on a phenotypic level, different mechanisms might be affecting the expression of these 
traits (Petelle et al., 2015). This is important because carryover effects (described by Sih et al., 2004) are a valid problem only if the two measures of the same trait in different contexts are indeed measures of the same trait, i.e., that the genetic correlation is approaching 1 . If there is no genetic correlation between these measures of the same trait, these measures should be seen as different traits, and selection could be acting independently on them. Second, if seemingly different traits in the same or different context have some overlap in the genomic structure, the evolution of these traits is not independent. This may lead to temporary constraints in the independent evolution of these traits (but see Roff et al., 1996).

\section{Molecular genetics of animal personality}

Knowledge of the molecular genetic basis of personality traits provides information on the expression and regulation of genes at the molecular level. Moreover, genes provide the foundation for the nervous system to express behaviours through the production of polypeptides. Knowing the structure and function of the molecular mechanisms in personality traits will help us in answering questions regarding the origin of the variation in personality traits, the expected responses to environmental variation, and the possible constraints there are in expressing behavioural variation. Identifying genetic variants in the genome will also elucidate the micro-evolutionary processes and historical selection behind personality traits, and will eventually help explain the maintenance of variation in personality (see also Bell \& Aubin-Horth, 2010). The rapid speed at which molecular genetic tools have been developed over the last decade has, in particular, helped to gain a more profound knowledge of the mechanisms by means of integrating quantitative genetics and molecular biology. 
Identifying the biological mechanisms of variation in behaviour can help us understand the genetic composition, characteristics of the nervous system, physiological state, developmental history, environmental factors and biochemical reactions an organism has and experiences during its lifetime (Anholt \& Mackay, 2009; Krebs \& Davies, 1997). Because of the complexity of personality and the multigenic architecture and high sensitivity to environmental influence, unravelling the genetic background of behaviour is challenging (Anholt \& Mackay, 2004; Bendesky \& Bargmann, 2011; Flint \& Mott, 2001; Flint, 2003).

The main resource for genetic variation is mutation. Often mutations are rare and neutral/deleterious but sometimes natural selection favors random mutations in the genome. If this mutation affects genes that control an organism's behaviour, this will cause the behavioural patterns to be altered. Genes that affect behaviour can have two kinds of effects. First, some genes influence the manifestation of the behaviour, i.e., the gene affects a trait in which there is little or no variation in the population, and the trait is not environmentally determined. This can be studied by mutagenesis approaches: targeted, gene-trap or ENU (N-ethyl-N- nitrosourea) mutagenesis, as it has been done, for example, in behavioural studies of mice (Belknap et al. 2001; Bucan \& Abel 2002; Vitaterna et al. 2006). In addition to these mutagenesis approaches and traditional transgenesis technologies, there are new genome editing techniques such as CRISPR-Cas-based RNA-guided DNA endonucleases (CRISPR-Cas) that allow genomes to be modified by targeted insertion, removal, or replacement of genomic DNA areas (reviewed by Kratochwil \& Meyer, 2014; Singh et al., 2015). In behavioural studies CRISPR-Cas has been used in mice. Swiech et 
al. (2014) perturbed multiple genes in the mouse brain in vivo by using the CRISPRCas method. These authors characterized the effects of genome modifications in postmitotic neurons using biochemical, genetic, electrophysiological, and behavioural readouts and found in behavioural tests that the modification affected the memory of the mice. Their study successfully demonstrated that the CRISPRCas method can be used in the studies of gene function in the brain.

For personality traits a more likely case is, when a particular set of genes contributes to the behavioural variation. With quantitative trait loci (QTL) mapping approaches (see examples below) it is possible to identify and estimate the contributions of these genes to the observed phenotypic variance (Boake et al., 2002). Any QTL analysis assumes that the behaviour is variable within the mapping population and that the effects of the QTL can be monitored by creating mutations, and this is possible especially in model organisms. However, it is often only possible to rely on the mutations that have occurred naturally and are manifesting in natural populations.

Behavioural genetics studies pose many problems that need to be resolved. One such problem in personality studies is that the replication of the behavioural measurements can be difficult. If the measured personality trait is relying on unreliable, non-replicated measures, this might give spurious results in, e.g., genetic association studies. However, some behaviours, like the reaction to novelty, can be measured only once. Another challenge is the small effect size (strength of the relationship between an individual genetic variant and personality) in association studies. Effect size determines the power of the study and it is dependent on the sample size. In many wild animal QTL studies, the sample sizes are in the hundreds 
(Slate, 2013) and the expectation is that increasing the sample size would help the QTL detection. This expectation has been proven correct in model organisms and in humans by Flint and Mackay (2009). However, even if the studies have small effect sizes, they can be useful. A single-nucleotide polymorphism (SNP) with a small effect may reveal the underlying biological pathways and especially when many of these small effect SNP studies are combined in meta-analyses, significant pathways may be revealed. For example, Ripke et al. (2013) used a multi-stage genome-wide association study (GWAS) that combined actual GWA analysis with a meta-analysis in a single study to reveal new risk loci for schizophrenia.

In this chapter we regard studies on wild species as studies in which the individuals under study are descended from recently sampled non-domesticated individuals. In contrast, genetic model species (i.e., model to human behaviour) are typically reared in the laboratory for many generations. Most behavioural genetic studies have been conducted on such genetic model organisms, which include mice, Drosophila, and, more recently, domesticated animals as subjects (Fitzpatrick et al., 2005; InoueMurayama, 2009). New models have also been emerging especially for behavioural studies. One of them being the honeybee, which have provided valuable insights into social behaviour (Fitzpatrick et al., 2005). Furthermore, the three-spined stickleback (Gasterosteus aculeatus) is a a well-known model species for evolutionary biology and it has a large amount of variation in behaviour as well as morphology and physiology (Bell \& Foster, 1994; Bell \& Stamps, 2004). Lastly, the great tit, Parus major, a wild passerine bird has proved to be an excellent species for ecological, evolutionary and behavioural studies (Laine et al., 2016). Despite the potential 
ecological and evolutionary significance of wild populations, and the fact that more variation in behaviour is seen in the natural setting, the underlying genetic basis of behaviours and especially personality traits has rarely been studied in free-living populations. However, there are methods available currently to change the situation. First, a 'candidate gene approach' can easily be used in wild populations (Fidler et al., 2007; Fitzpatrick et al., 2005). Second, the most recent developments in genomic techniques enable genome-wide approaches in QTL analyses to be undertaken in almost any species. The lack of genome sequence information has been one of the main limitations of conducting both candidate gene and QTL studies in non-model organisms but, thanks to technological developments, more and more whole genomes for species have become available. In the following paragraphs we present examples from both candidate gene and QTL approaches.

\section{a. Candidate gene approaches}

In the candidate gene approach the assumption is that the trait is determined to a significant extent by a smaller number of genes, whose effects are large enough to be detected. Many recent studies that use a candidate gene approach, have demonstrated that the knowledge of gene-trait associations in "model" organisms such as humans, domestic fowl or mice, can be utilized for studying the genetic basis of behavioural variation in wild animal populations (Fidler et al., 2007; Fitzpatrick et al., 2005). In addition to taking candidate genes from model organism studies, genes can be selected based on an a prior knowledge of the biochemical pathway in the trait in question. For example, a zebrafish model was used successfully to identify many candidate genes and pathways to regulating 
aggression in fish, combining gene expression profiling, behavioural analyses, and pharmacological manipulations (Filby et al., 2010).

In a candidate gene approach wild individuals can be categorized into behavioural types without measuring the actual behaviour. In addition, candidate gene studies require little prior sequence information and are therefore well-suited for behavioural genetics analyses in natural populations of non-model species (Fitzpatrick et al., 2005). Earlier studies demonstrated that molecular markers located within or near candidate genes can be a useful resource for the identification of genes associated with adaptive phenotypic divergence, especially when there are no other genomic resources available (Shikano et al., 2010; Tonteri et al., 2010). In addition, markers closely linked to functionally important genes are useful in the construction of comparative genetic maps, in which they can be used as comparative anchor-tagged sequence loci (Lyons et al., 1997).

In humans, among the most studied candidate genes for personality variation are various polymorphisms within the dopamine receptor D4 (DRD4) gene and the serotonin transporter gene, solute carrier family 6 member 4 (SLC6A4/SERT) (Delvecchio et al., 2016; Savitz \& Ramesar, 2004). Polymorphisms within the DRD4 gene have been found to account for about $3 \%$ of the variation in novelty seeking in humans (Munafò et al., 2008). There are a series of polymorphic regions that might affect the expression or function of serotonin transporter (Ozaki et al., 2003; Wendland et al., 2006). The most studied polymorphism is the 5-HTTLPR, which is a functional polymorphism within the promoter sequence of the serotonin transporter gene (Heils et al., 1995), and it has been linked to variety of behaviours and 
behavioural disorders, including impulsivity, mood, and anxiety (Gordon \& Hen, 2004; Eley \& Plomin, 1997). However, the evidence for an association is inconsistent when slightly different measures of the trait (harm avoidance, neuroticism, etc.) are used (Munafò et al., 2009). Nevertheless, SLC6A4 may play a role in anxiety and other affective disorders, but its effects might be subtle, for example, on amygdala activation, which has been associated with depression and anxiety (Murphy et al., 2013).

In addition to in humans, an association between exploratory behaviour and the DRD4 homologue has been detected in many species, for example, in apes (Shimada et al., 2004), dogs (Ito et al., 2004), fish (Boehmler et al., 2007), and birds (Fidler et al., 2007). In the latter study, Fidler et al. (2007) found that great tits artificially selected for divergent levels of exploratory behaviour differed in the allele frequency of an exonic SNP in the DRD4 gene. The association was confirmed in a natural population, where the levels of exploratory behaviour differed for great tits with different genotypes (Fidler et al., 2007). However, the DRD4 studies done with great tits also highlight concerns regarding candidate gene studies: (unaccounted) population structuring leading to possible false positive associations especially in the case of complex traits like personality. When the association was tested across samples of four great tit populations, the association was significant in only one with an estimated effect size of around $5 \%$ (Korsten et al., 2010). This could indicate that the DRD4 polymorphism is linked to the functional variant in some but not all populations, or that the association is dependent on the environment or other population-specific characteristics (Korsten et al., 2010; Mueller et al., 2013). 
Another popular gene that has been used in personality studies is the monoamine oxidase $\mathrm{A}(M A O-A)$, polymorphisms of which have been linked to antisocial behavior and aggression in humans and mice (Nelson \& Trainor, 2007; Shih \& Thompson, 1999; Scott et al., 2008). One well-known example comes from human studies where the interaction between the $M A O-A$ genotype and the rearing environment affected the aggressive behaviour (Caspi et al. 2002). This study showed that children carrying the short form of the MAO-A promoter gene, which decreases MAO-A activity, are more likely to develop antisocial problems when exposed to abusive home environments. This environmental effect has less effect on individuals carrying the long form of the promoter. Later studies supported these results by showing that the low activity $M A O-A$ in combination with traumatic early life events predisposes individuals toward aggressive behaviour (Frazetto et al., 2007; KimCohen et al., 2006) and in a recent review of Weeland et al. (2015) they describe ways to conduct and compare gene-environment interactions in mental disorders. Other genes with possible effects on variation in personality include the dopamine receptor D2 gene, the serotonin receptor genes 5-HT2c and HTR2a, and the tyrosine hydroxylase gene (reviewed in Savitz \& Ramesar, 2004).

In addition to population structuring affecting the candidate gene studies, there are limitations to the use of candidate gene studies for animal personality. Where this approach directly tests the effects of mutations located in specific genes for their association to the trait of interest, it can permit only the identification of genes that have been, in some way, related to the trait previously. The candidate gene approach, therefore, cannot reveal the association of totally new mutations, unlike genome-wide approaches, such as linkage mapping or genome-wide association 
studies. Moreover, because candidate gene studies are often conducted with relatively small samples, only polymorphisms that are relatively abundant in the population can be tested. Furthermore, it is likely that many genes are involved in epistatic interactions. The effects of single polymorphism or gene associations, might therefore be a bad indicator for the ultimate role of the genes for variation in trait expression. Thus, the results from candidate gene studies need to be validated by testing for these associations in several populations and species, and also by adding gene expression and genome-wide association approaches to ascertain causal links between the polymorphism and the behaviour.

\section{b. Genome-wide QTL approaches}

Another approach to assess which genomic regions underlie variation in personality is a more bottom-up approach in which no a priori expectation about genes related to measured traits is needed by using QTL analyses. This approach is more suitable for studies of behavior because of the multigenic architecture of behavioural traits. These genome-wide QTL analyses can be divided into two groups: linkage based studies and association mapping studies.

\section{i. Linkage based mapping (QTL mapping)}

In linkage based mapping, also known as QTL mapping, the aim is to identify genetic markers that predict the phenotype, by linking pedigreed populations with individual genotypic data consisting of genetic markers that are evenly distributed over the whole genome (Slate, 2005). This analysis method is based on pedigree information (one or many pedigrees from natural populations or crossing experiments) and the 
mapping populations consist either of inbred line crosses, crosses between outbred populations, or natural populations with known pedigree structure (Lynch \& Walsh, 1998; Slate, 2005).

The predictive markers are close to the causal loci, and so the predictive markers and the causal loci tend to segregate together. Most predictive markers are expected to be in close proximity to the causal locus because the probability of recombination breaking the linkage increases with physical distance. Linkage based mapping permits the determination of genomic regions that are involved in explaining heritable phenotypic variation and this is then used to predict the genetic architecture of the measured trait (Erickson et al., 2004). Often the result is a candidate chromosomal region linked to variation in the phenotype, which covers several dozens to hundreds of genes. This region can be narrowed by haplotype sharing or the identification of more polymorphic markers at these specific sites by using so called fine-mapping. The candidate genes are identified from these areas for further genetic studies with the aim of identifying loci that are of major importance for the trait variation.

Many QTL mapping studies on animal personality traits have been conducted with rodents by using controlled crosses between lines or strains (e.g. Gershenfeld et al., 1997; Hovatta \& Barlow, 2008). Additionally, personality has been found to be associated with animal well-being in farm animals (Christiansen \& Forkman, 2007; Koolhaas et al., 2001; Rodenburg et al., 2008), leading to behaviour genetic studies identifying QTL for behavioural traits in livestock (Haskell et al., 2014). For example, in a study by Gutierrez-Gil et al. (2008) 29 QTL regions were identified in a cross between two cattle populations measured for several temperament related 
behaviours. In total, each region explained only a small fraction of the phenotypic variation, ranging from 4 to 8 per cent. However, the most notable candidate gene found in one of the regions was the $D R D 4$ gene, showing that $D R D 4$ is probably an important gene in cattle personality traits.

In 2005, Slate published a review of the prospects for QTL studies in natural populations. This paper has good guidelines for what techniques can be used to perform QTL studies with non-model organisms, especially in the context of ecological and evolutionary issues. The biggest issue for most species for which personality data have been collected in natural populations, is the lack of pedigree information and the lack of a sufficient number of markers to be able to construct genetic maps. It is therefore possible that some QTL mapping studies on behaviour did not result in the detection of any QTL and thus remained unpublished, even in the presence of heritable variation. Since modern meta-analytical methods could overcome these biases, there is an urge to also publish studies when no significant QTLs are found. However, genomic polymorphism data for high-quality genetic maps are now becoming available for many non-model species. A whole-genome linkage map of the zebra finch (Taeniopygia guttata) based on about 2000 SNP markers has been constructed (Backström et al., 2010), while tens of thousands of SNPs have been used to construct the great tit linkage map (Van Bers et al., 2010; van Oers et al., 2015) to name just two dominant species used in animal personality research.

Nevertheless, association studies in wild populations based on these numbers of markers show only limited success. In a QTL study on personality in wild bighorn sheep (Ovis canadensis) Poissant et al. (2013) identified two suggestive QTLs using 
a linkage analysis based on 238 microsatellite loci genotyped in 310 pedigreed individuals, but no regions exceeding genome-wide significance were detected. A study on exploratory behaviour in great tits in two independent replicate populations that used both linkage and association mapping methods also failed to reveal any significant regions associated with this often investigated personality trait (Santure et al., 2015), even though sample sizes were larger than in the wild bighorn sheep study (1000 individuals and 5500 SNPs).

To increase the power of these QTL studies, natural variation can be magnified by artificial selection or artificial crosses, by, for example, F2-cross designs. In Laine et al. (2014) nine-spined stickleback (Pungitius pungitius) study, three behavioural measurements were QTL mapped using a F2-cross design between marine and pond populations. Many QTL regions were located in the linkage map, which supports the polygenic regulation of behaviour. In one of the regions they found QTLs influencing different behavioural traits. This finding suggests that the genetic factors influencing one behaviour may have pleiotropic effects on other behaviours or that genetic factors influencing different behaviours cluster into this linkage group. QTL mapping of schooling behaviour has also been successfully used in threespined sticklebacks by using two types of crosses: benthic $\times$ marine backcrosses and an F2 intercross, which revealed new candidate regions for behavioural differences in sticklebacks (Greenwood et al., 2015). Another example of QTL mapping and cross design comes from the study of anti-predatory behaviour in rainbow trout (Oncorhynchus mykiss) (Christensen et al., 2014). Through using clonal rainbow trout lines derived from either wild or hatchery populations they 
identified several behaviours that varied between clonal lines and they found QTLS for behavioural and size traits.

\section{ii. Association mapping}

Association mapping, also known as genome-wide association study (GWAS), is also based on recombination, but the recombination used in this method is historical. GWAS uses population-based data with abundant recombination history and individuals are genotyped with thousands of SNPs by using microarrays or chipsequencing to capture most of the genetic variation in the studied population (Risch 2000). With this method, the entire genome is covered and the aim is to identify the genes with potentially known functions that are causing variation in the trait of interest (Bush and Moore, 2012). The significant genomic areas or candidate SNPs can be used for further fine mapping or in experimental studies. The power to detect QTL at an intermediate frequency is comparable between linkage and GWA studies. However, in GWA studies, allele frequencies can be more extreme and this means that increasing sample sizes and also marker densities increases the number of QTL detected and this decreases the average effect sizes (Mackay et al., 2009).

The vast majority of personality GWA studies use human participants because of the high numbers of individuals and markers needed for the analyses (De Moor et al. 2009). The first GWA study on the five major human personality factors: neuroticism, extraversion, agreeableness, openness to experience, and conscientiousness, used a sample of 3972 individuals from an isolated population on Sardinia, Italy that was genotyped with 362129 SNPs (Terracciano et al. 2008). A few of the most promising SNPs that were identified were successfully replicated in two independent samples, 
one from USA and the other from the Netherlands. The authors highlight two important conclusions that can be drawn from this pioneering study. First, personality traits are influenced by many genes that each explain only small proportion of variation (1-2\%) and these polymorphisms are detected only when sample sizes are large enough. Second, genetic effects are most probably found when specific phenotypes are measured, rather than when pooled into broader factors or by using principal component analysis (Terracciano et al., 2008). This suggests that personality traits, like boldness or risk-taking behaviours, should be broken down into smaller units of individual behaviours. The same outcome could also indicate that specific genes play a role in determining variation in single personality traits, but additional genes modify the correlations among traits. This issue points to one of the challenges in the behavior genetics of personality is the definition and quantification of behaviour (Sokolowski, 2001). We expect that more association studies will be possible in natural systems in the near future, because with decreasing sequencing and genotyping costs and the invention of new genomic and statistical tools, these methods will become available also for non-model species as in Santure et al. (2015).

\section{Pleiotropy}

Generally, no genes have been identified as solely affecting behaviour, and "behavioural genes" therefore do not directly control behaviour. These genes instead influence the development and function of behaviour by affecting the development of the nervous system that is related to adult behaviour (Sokolowski, 2001). In addition, these genes affect behaviour, physiology, and morphology. Genes, therefore, are 
often responsible for many distinct and unrelated phenotypes, a phenomenon called pleiotropy (Stearns, 2010). Pleiotropic effects in behaviour have especially been studied in insects. In the fruit fly (Drosophila melanogaster) system where mutants are relatively easy to produce, researchers discovered that producing mutants in candidate genes for behaviour had pleiotropic effects in both development and behaviour, or in several behaviours (reviewed by Anholt \& Mackay, 2004 and Sokolowski, 2001). Furthermore, in honeybees (Apis mellifera) there have been demonstrations of pleiotropy in the context of reproduction and social behaviour (reviewed by Page et al., 2012). But examples extend also to vertebrates. In the guppy (Poecilia retucilata), lines selected for large or for small brains, were known to differ in cognitive ability (Kotrschal et al., 2014). When testing individuals from these selected lines for three personality traits, the authors found that large-brained animals explored an open field faster and stayed in the open more compared to individuals from the small-brained line. Large-brained animals also secreted less cortisol in a stressful situation and were slower to feed from a novel food source, altogether pointing towards a more reactive personality type in large-brained animals, indicating pleiotropic effects between brain development and behaviour.

In a combined QTL and eQTL study on a range of behavioural traits in 294 German Landrace piglets (Sus domesticus), the most evident gene found was PER1, which has known physiological implications for circadian rhythms, was shown to associate with variation in a backtest trait, a validated personality and coping trait in pigs (Ponsuksili et al., 2015), indicating that this gene may have a role for both timing and personality traits. Another striking example of pleiotropic effects between physiology 
and behaviour comes from the melanocortin system studies. It has been shown that there is a widespread relationship between melanin-based coloration and other phenotypic traits in vertebrates (reviewed by Ducrest et al., 2008). One of the most famous examples of pleiotropism comes from the domestication of wild silver foxes (Vulpes vulpes). The Russian geneticist Belyaev found that, when selecting for tame foxes, many other features were also changed such as droopy ears, a smaller skull size, bigger litter sizes, a faster reproductive cycle, and a piebald fur pattern (white patches, depigmentation) (Trut et al., 1997; Trut, 1999). More recent examples of this relationship include Mafli, Wakamatsu, and Roulin (2011) who found that darker shell coloration predicted greater aggressiveness and boldness in Hermann's tortoises (Eurotestudo boettgeri). Genetic studies should therefore not only concentrate on the most popular genes studied in behavioural genetics but widen the search to other gene-networks as well.

\section{Future research}

Research on the genetic mechanism of personality differences saw a transition from testing effects of candidate gene variation towards whole genome methods in molecular genetic. For quantitative genetics it changed from describing heritability components in unmanipulated mid-term pedigrees to multivariate experimental setups. Now there is a great need for integrating such molecular and quantitative genetic methods (Bell \& Dochtermann 2015). For a truly integrative framework, experiments should be planned that allow variance partitioning at the level of both trait variation and trait plasticity. A main challenge will be to link the heritable variation, or even the variance components responsible for heritable variation, to 
genomic mechanisms. Moreover, the conceptual frameworks developed in quantitative and molecular genetics should be integrated, to develop testable hypotheses.

Because genes and environment cannot be separated (see e.g., Groothuis \& Trillmich, 2011; van Oers et al., 2005), future research also needs to focus on integrating genetic research with epigenetic research. In addition to additive genetic variation and dominance genetic effects, heritable personality differences can arise from transgenerational epigenetic effects, i.e., all transgenerational processes that contribute to the non-genetic determination of the phenotype, such as paternal/maternal effects and permanent environmental effects (Youngson \& Whitelaw, 2008). The genomic mechanisms responsible such transgenerational effects are largely unknown, although processes such as DNA methylation of cytosines, small RNA's and the modification of chromatin proteins are strong candidates (Moore 2016). Therefore, epigenetic mechanisms may play a major role in the expression and heritability of animal personality traits (Ledón-Rettig et al., 2012). First, studies that investigate the DNA methylation in animals indicate that DNA methylation might be a mechanism involved in mediating transgenerational inheritance. In a study of great tits in the Netherlands, birds from a line selected for fast exploratory behaviour (Drent et al., 2003), have been found to have increased levels of DNA methylation in the promoter region of DRD4 (Verhulst et al., 2016). This study was confirmed by results in a wild population in Spain, where methylation at a single $\mathrm{CpG}$ dinucleotide in the $D R D 4$ promoter region was related to exploration score in urban great tits (Riyahi et al., 2015). These results support the idea that 
some of the effects found for DRD4 may be mediated by DNA methylation (Verhulst et al., 2016). The next steps should focus on investigating the contribution of epigenetic processes, such as DNA methylation for personality traits in natural systems and its subsequent role for understanding the ecology and evolution of behavioural consistency.

Where current studies focus on finding allelic differences among individuals that are associated with individual differences in behaviour, in future studies we will be moving towards more concrete functional links between genomic variation, gene expression, and behaviour. A very nice example of this in a recent study is a largescale genetical genomics analysis of chicken (Gallus gallus) brains from individuals differing in open field behaviour (Johnson et al., 2016). By combining information on genome-behaviour associations with associations between gene-expression and behaviour, the authors identified 10 putative loci that correlated with both behaviour (bQTL) and gene expression (eQTL). Four of these loci were formerly linked to bipolar disorder, major depressive disorder, and schizophrenia in the mouse Heterogeneous Stock anxiety data set and human GWAS data sets. Assuming that most adaptive and functional variation lies in the coding sequence of genes underestimates the role of regulatory mechanisms (Rasmuson, 2009; Groothuis \& Trillmich, 2011). Studying gene expression with gene polymorphisms rather than a single one in isolation will be more likely to lead to further understanding the consistency and plasticity of personality traits.

The validation of the findings of association studies will be mitigated by new tools that are increasingly becoming available. Tools, that allow for experimental 
modification of genomes, even in unconventional model species such as wild species, make personality genetics research more and more experimental. One example of such a tool is the above-mentioned CRISP-R, which has many advantages for behavioural studies. For one, this method allows many mutations to be introduced in parallel, which is especially advantageous for multi-genic behavioural traits. In addition, CRISP-R can be applied to any species.

\section{Conclusion}

In the past two decades, research on the genetical background of animal personality traits focussed on mapping the quantitative and molecular structure of personality traits, trait correlations, and plasticity. We should now move towards more integration among fields of research. Animal personality offers a unique opportunity to not only combine quantitative genetics with molecular genetics, it also offers the greatest diversity in processes to be studied in concordance, in single organisms. Animal personality traits as labile quantitative traits, are playgrounds for studying intraindividual, inter-individual and inter-trait associations. Personality will therefore help us understand the complexity of organisms at different levels. Genetic studies may thereby function as a multidisciplinary link between research fields in achieving a more holistic understanding how organisms react to their changing environments. 


\section{References}

Adams, M. J., King, J. E., \& Weiss, A. (2012). The Majority of Genetic Variation in Orangutan Personality and Subjective Well-Being is Nonadditive. Behavior Genetics, 42(4), 675-686. doi:10.1007/s10519-012-9537-y

Anholt, R. R., \& Mackay, T. F. (2009). Principles of behavioral genetics. Amsterdam: Academic.

Anholt, R. R., \& Mackay, T. F. (2004). Quantitative genetic analyses of complex behaviours in Drosophila. Nature Reviews Genetics, 5(11), 838-849. doi:10.1038/nrg1472

Arnold, K. E., Ramsay, S. L., Donaldson, C., \& Adam, A. (2007). Parental prey selection affects risk-taking behaviour and spatial learning in avian offspring. Proceedings of the Royal Society B: Biological Sciences, 274(1625), 25632569. doi:10.1098/rspb.2007.0687

Backstrom, N., Forstmeier, W., Schielzeth, H., Mellenius, H., Nam, K., Bolund, E., . . . Ellegren, H. (2010). The recombination landscape of the zebra finch Taeniopygia guttata genome. Genome Research, 20(4), 485-495. doi:10.1101/gr.101410.109

Bell, A. M., \& Aubin-Horth, N. (2010). What can whole genome expression data tell us about the ecology and evolution of personality? Philosophical Transactions of the Royal Society B: Biological Sciences, 365(1560), 4001-4012. doi:10.1098/rstb.2010.0185

Bell, A. M., \& Stamps, J. A. (2004). Development of behavioural differences between individuals and populations of sticklebacks, Gasterosteus aculeatus. Animal Behaviour, 68(6), 1339-1348. doi:10.1016/j.anbehav.2004.05.007 
Bell, A. M., \& Dochtermann, N. A. (2015). Integrating molecular mechanisms into quantitative genetics to understand consistent individual differences in behavior. Current Opinion in Behavioral Sciences, 6, 111-114. doi:10.1016/j.cobeha.2015.10.014

Bell, M. A., \& Foster, S. A. (1994). The evolutionary biology of the threespine stickleback. Oxford: Oxford University Press.

Belknap, J. K., Hitzemann, R., Crabbe, J. C., Phillips, T. J., Buck, K. J., \& Williams, R. W. (2001). QTL analysis and genomewide mutagenesis in mice: complementary genetic approaches to the dissection of complex traits. Behavior Genetics, 31(1), 5-15. doi: 10.1023/A:1010249607128

Bendesky, A., \& Bargmann, C. I. (2011). Genetic contributions to behavioural diversity at the gene-environment interface. Nature Reviews Genetics. doi:10.1038/nrg3065

Bers, N. E., van Oers, K., Kerstens, H. H., Dibbits, B. W., Crooijmans, R. P., Visser, M. E., \& Groenen, M. A. (2010). Genome-wide SNP detection in the great tit Parus major using high throughput sequencing. Molecular Ecology, 19, 89-99. doi:10.1111/j.1365-294x.2009.04486.x

Boake, C., Arnold, S., Breden, F., Meffert, L., Ritchie, M., Taylor, B., ... Moore, A. (2002). Genetic Tools for Studying Adaptation and the Evolution of Behavior. The American Naturalist, 160(S6). doi:10.1086/342902

Boehmler, W., Carr, T., Thisse, C., Thisse, B., Canfield, V. A., \& Levenson, R. (2007). D4 Dopamine receptor genes of zebrafish and effects of the antipsychotic clozapine on larval swimming behaviour. Genes, Brain and Behavior, 6(2), 155-166. doi:10.1111/j.1601-183x.2006.00243.x 
Brem, R. B., \& Kruglyak, L. (2005). The landscape of genetic complexity across 5,700 gene expression traits in yeast. Proceedings of the National Academy of Sciences, 102(5), 1572-1577. doi:10.1073/pnas.0408709102

Brommer, J. E., \& Kluen, E. (2012). Exploring the genetics of nestling personality traits in a wild passerine bird: Testing the phenotypic gambit. Ecology and Evolution, 2(12), 3032-3044. doi:10.1002/ece3.412

Brydges, N. M., Colegrave, N., Heathcote, R. J., \& Braithwaite, V. A. (2008). Habitat stability and predation pressure affect temperament behaviours in populations of three-spined sticklebacks. Journal of Animal Ecology, 77(2), 229-235. doi:10.1111/j.1365-2656.2007.01343.x

Bućan, M., \& Abel, T. (2002). The Mouse: Genetics Meets Behaviour. Nature Reviews Genetics, 3(2), 114-123. doi:10.1038/nrg728

Burtt, H.E., Giltz, M.L. (1973). Personality as a Variable in the Behavior of Birds. Journal of Science, 73(2), 65-82

Careau, V., Thomas, D., Pelletier, F., Turki, L., Landry, F., Garant, D., \& Réale, D. (2011). Genetic correlation between resting metabolic rate and exploratory behaviour in deer mice (Peromyscus maniculatus). Journal of Evolutionary Biology, 24(10), 2153-2163. doi:10.1111/j.1420-9101.2011.02344.x

Carere, C., \& Maestripieri, D. (2013). Animal personalities: Behavior, physiology, and evolution. Chicago: The University of Chicago Press.

Caspi, A. (2002). Role of Genotype in the Cycle of Violence in Maltreated Children. Science, 297(5582), 851-854. doi:10.1126/science.1072290

Christensen, K. A., Brunelli, J. P., Wheeler, P. A., \& Thorgaard, G. H. (2014). Antipredator Behavior QTL: Differences in Rainbow Trout Clonal Lines Derived 
from Wild and Hatchery Populations. Behavior Genetics, 44(5), 535-546. doi:10.1007/s10519-014-9663-9

Christiansen, S. B., \& Forkman, B. (2007). Assessment of animal welfare in a veterinary context-A call for ethologists. Applied Animal Behaviour Science, 106(4), 203-220. doi:10.1016/j.applanim.2007.01.004

Class, B., \& Brommer, J. E. (2015). A strong genetic correlation underlying a behavioural syndrome disappears during development because of genotypeage interactions. Proceedings of the Royal Society B: Biological Sciences, 282(1809), 20142777. doi:10.1098/rspb.2014.2777

Danchin, É, Charmantier, A., Champagne, F. A., Mesoudi, A., Pujol, B., \& Blanchet, S. (2011). Beyond DNA: Integrating inclusive inheritance into an extended theory of evolution. Nature Reviews Genetics, 12(7), 475-486. doi:10.1038/nrg3028

Davies, N. B., \& Krebs, J. R. (1984). Behavioural ecology: An evolutionary approach. Oxford: Blackwell.

Delvecchio, G., Bellani, M., Altamura, A. C., \& Brambilla, P. (2016). The association between the serotonin and dopamine neurotransmitters and personality traits. Epidemiology and Psychiatric Sciences, 25(02), 109-112. doi:10.1017/s2045796015001146

De Moor, Costa, P. T., Terracciano, A., Krueger, R. F., de Geus, E. J. C., Toshiko, T., ... Boomsma, D. I. (2012). Meta-analysis of genome-wide association studies for personality. Molecular Psychiatry, 17(3), 337-349. doi:10.1038/mp.2010.128.Meta-analysis 
Dingemanse, N. J., Plas, F. V., Wright, J., Reale, D., Schrama, M., Roff, D. A., . . Barber, I. (2009). Individual experience and evolutionary history of predation affect expression of heritable variation in fish personality and morphology. Proceedings of the Royal Society B: Biological Sciences, 276(1660), 12851293. doi:10.1098/rspb.2008.1555

Dingemanse, N. J., \& Wolf, M. (2013). Between-individual differences in behavioural plasticity within populations: Causes and consequences. Animal Behaviour, 85(5), 1031-1039. doi:10.1016/j.anbehav.2012.12.032

Dingemanse, N. J., \& Dochtermann, N. A. (2012). Quantifying individual variation in behaviour: Mixed-effect modelling approaches. Journal of Animal Ecology, 82(1), 39-54. doi:10.1111/1365-2656.12013

Dochtermann, N. A., \& Roff, D. A. (2010). Applying a quantitative genetics framework to behavioural syndrome research. Philosophical Transactions of the Royal Society B: Biological Sciences, 365(1560), 4013-4020. doi:10.1098/rstb.2010.0129

Dochtermann, N. A., Schwab, T., \& Sih, A. (2014). The contribution of additive genetic variation to personality variation: Heritability of personality. Proceedings of the Royal Society B: Biological Sciences, 282(1798), 20142201-20142201. doi:10.1098/rspb.2014.2201

Drent, P. J., van Oers, K., \& Noordwijk, A. J. (2003). Realized heritability of personalities in the great tit (Parus major). Proceedings of the Royal Society B: Biological Sciences, 270(1510), 45-51. doi:10.1098/rspb.2002.2168 
Ducrest, A., Keller, L., \& Roulin, A. (2008). Pleiotropy in the melanocortin system, coloration and behavioural syndromes. Trends in Ecology \& Evolution, 23(9), 502-510. doi:10.1016/j.tree.2008.06.001

Eaves, L., \& Verhulst, B. (2014). Problems and Pit-Falls in Testing for $G \times E$ and Epistasis in Candidate Gene Studies of Human Behavior. Behavior Genetics, 44(6), 578-590. doi:10.1007/s10519-014-9674-6

Eley, T. C., \& Plomin, R. (1997). Genetic analyses of emotionality. Current Opinion in Neurobiology, 7(2), 279-284. doi:10.1016/s0959-4388(97)80017-7

Erickson, D. L., Fenster, C. B., Stenoien, H. K., \& Price, D. (2004). Quantitative trait locus analyses and the study of evolutionary process. Molecular Ecology, 13(9), 2505-2522. doi:10.1111/j.1365-294x.2004.02254.x

Falconer, D., \& Mackay, T. F. C. (1996). Introduction to Quantitative Genetics (4th editio.). Longman.

Fidler, A. E., van Oers, K., Drent, P. J., Kuhn, S., Mueller, J. C., \& Kempenaers, B. (2007). Drd4 gene polymorphisms are associated with personality variation in a passerine bird. Proceedings of the Royal Society B: Biological Sciences, 274(1619), 1685-1691. doi:10.1098/rspb.2007.0337

Filby, A. L., Paull, G. C., Hickmore, T. F., \& Tyler, C. R. (2010). Unravelling the neurophysiological basis of aggression in a fish model. BMC Genomics, 11(1), 498. doi:10.1186/1471-2164-11-498

Fitzpatrick, M., Benshahar, Y., Smid, H., Vet, L., Robinson, G., \& Sokolowski, M. (2005). Candidate genes for behavioural ecology. Trends in Ecology \& Evolution, 20(2), 96-104. doi:10.1016/j.tree.2004.11.017 
Flint, J., \& Mackay, T. F. (2009). Genetic architecture of quantitative traits in mice, flies, and humans. Genome Research, 19(5), 723-733. doi:10.1101/gr.086660.108

Flint, J. (2002). Analysis of quantitative trait loci that influence animal behavior. Journal of Neurobiology, 54(1), 46-77. doi:10.1002/neu.10161

Flint, J., \& Mott, R. (2001). Finding The Molecular Basis Of Quantitative Traits: Successes And Pitfalls. Nature Reviews Genetics, 2(6), 437-445. doi:10.1038/35076585

Frazzetto, G., Lorenzo, G. D., Carola, V., Proietti, L., Sokolowska, E., Siracusano, A., . . Troisi, A. (2007). Early Trauma and Increased Risk for Physical Aggression during Adulthood: The Moderating Role of MAOA Genotype. PLoS ONE, 2(5). doi:10.1371/journal.pone.0000486

Gershenfeld, H. K., \& Paul, S. M. (1997). Mapping Quantitative Trait Loci for Fearlike Behaviors in Mice. Genomics, 46(1), 1-8. doi:10.1006/geno.1997.5002 Gordon, J. A., \& Hen, R. (2004). Genetic Approaches To The Study Of Anxiety. Annual Review of Neuroscience, 27(1), 193-222. doi:10.1146/annurev.neuro.27.070203.144212

Greenwood, A. K., Ardekani, R., Mccann, S. R., Dubin, M. E., Sullivan, A., Bensussen, S., . . . Peichel, C. L. (2015). Genetic Mapping of Natural Variation in Schooling Tendency in the Threespine Stickleback. G3\&amp;\#58; Genes/Genomes/Genetics, 5(5), 761-769. doi:10.1534/g3.114.016519 Groothuis, T. G., \& Trillmich, F. (2011). Unfolding personalities: The importance of studying ontogeny. Developmental Psychobiology, 53(6), 641-655. doi:10.1002/dev.20574 
Guan, Y., \& Stephens, M. (2011). Bayesian variable selection regression for genome-wide association studies and other large-scale problems. The Annals of Applied Statistics, 5(3), 1780-1815. doi:10.1214/11-aoas455

Gutierrez-Gil, B., Ball, N., Burton, D., Haskell, M., Williams, J. L., \& Wiener, P. (2008). Identification of Quantitative Trait Loci Affecting Cattle Temperament. Journal of Heredity, 99(6), 629-638. doi:10.1093/jhered/esn060

Haskell, M. J., Simm, G., \& Turner, S. P. (2014). Genetic selection for temperament traits in dairy and beef cattle. Frontiers in Genetics, 5. doi:10.3389/fgene.2014.00368

Heils, A., Teufel, A., Petri, S., Seemann, M., Bengel, D., Balling, U., . . Lesch, K. -. $(1995,10)$. Functional promoter and polyadenylation site mapping of the human serotonin (5-HT) transporter gene. Journal of Neural Transmission, 102(3), 247-254. doi:10.1007/bf01281159

Henderson, C. R. (1984). Applications of linear models in animal breeding. Guelph, Ont.: University of Guelph.

Hovatta, I., \& Barlow, C. (2008). Molecular genetics of anxiety in mice and men. Annals of Medicine, 40(2), 92-109. doi:10.1080/07853890701747096 Inoue-Murayama, M. (2009). Genetic polymorphism as a background of animal behavior. Animal Science Journal, 80(2), 113-120. doi:10.1111/j.17400929.2008.00623.x

Ito, H., Nara, H., Inoue-Murayama, M., Shimada, M. K., Koshimura, A., Ueda, Y., . . . Ito, S. (2004). Allele Frequency Distribution of the Canine Dopamine Receptor D4 Gene Exon III and I in 23 Breeds. Journal of Veterinary Medical Science, 66(7), 815-820. doi:10.1292/jvms.66.815 
Johnsson, M., Williams, M. J., Jensen, P., \& Wright, D. (2016). Genetical Genomics of Behavior: A Novel Chicken Genomic Model for Anxiety Behavior. Genetics, 202(1), 327-340. doi:10.1534/genetics.115.179010

Jordan, K. W., Carbone, M., Yamamoto, A., Morgan, T. J., \& Mackay, T. F. (2007). Quantitative genomics of locomotor behavior in Drosophila melanogaster. Genome Biology, 8(8). doi:10.1186/gb-2007-8-8-r172

Kim-Cohen, J., Caspi, A., Taylor, A., Williams, B., Newcombe, R., Craig, I. W., \& Moffitt, T. E. (2006). MAOA, maltreatment, and gene-environment interaction predicting children's mental health: New evidence and a meta-analysis. Molecular Psychiatry, 11(10), 903-913. doi:10.1038/sj.mp.4001851 Koolhaas, J.M., De Boer, S.F., Buwalda, B., Van der Vegt, B.J., Carere, C. \& Groothuis, A.G.G. (2001). How and why coping systems vary among individuals. In Coping with challenge: welfare in animals including humans (ed. D. M. Broom), pp. 197-209. Dahlem, Berlin: Dahlem University Press.

Korsten, P., Mueller, J. C., Hermannstädter, C., Bouwman, K. M., Dingemanse, N. J., Drent, P. J., . . Kempenaers, B. (2010). Association between DRD4 gene polymorphism and personality variation in great tits: A test across four wild populations. Molecular Ecology, 19(4), 832-843. doi:10.1111/j.1365$294 x .2009 .04518 . x$

Kotrschal, A., Lievens, E. J., Dahlbom, J., Bundsen, A., Semenova, S., Sundvik, M., . .. Kolm, N. (2014). Artificial Selection On Relative Brain Size Reveals A Positive Genetic Correlation Between Brain Size And Proactive Personality In The Guppy. Evolution, 68(4), 1139-1149. doi:10.1111/evo.12341 
Kratochwil, C. F., \& Meyer, A. (2014). Closing the genotype-phenotype gap:

Emerging technologies for evolutionary genetics in ecological model vertebrate systems. BioEssays, 37(2), 213-226. doi:10.1002/bies.201400142

Kruuk, L. E. (2004). Estimating genetic parameters in natural populations using the 'animal model' Philosophical Transactions of the Royal Society B: Biological Sciences, 359(1446), 873-890. doi:10.1098/rstb.2003.1437

Kruuk, L. E., Slate, J., \& Wilson, A. J. (2008). New Answers for Old Questions: The Evolutionary Quantitative Genetics of Wild Animal Populations. Annual Review of Ecology, Evolution, and Systematics, 39(1), 525-548. doi:10.1146/annurev.ecolsys.39.110707.173542

Laine, V. N., Gossmann, T. I., Schachtschneider, K. M., Garroway, C. J., Madsen, O., Verhoeven, K. J., . . . Groenen, M. A. (2016). Evolutionary signals of selection on cognition from the great tit genome and methylome. Nature Communications, 7, 10474. doi:10.1038/ncomms10474

Laine, V. N., Herczeg, G., Shikano, T., Vilkki, J., \& Merilä, J. (2013). QTL Analysis of Behavior in Nine-Spined Sticklebacks (Pungitius pungitius). Behavior Genetics, 44(1), 77-88. doi:10.1007/s10519-013-9624-8

Ledon-Rettig, C. C., Richards, C. L., \& Martin, L. B. (2012). Epigenetics for behavioral ecologists. Behavioral Ecology, 24(2), 311-324. doi:10.1093/beheco/ars145

Lynch, M., \& Walsh, B. (1998). Genetics and analysis of quantitative traits. Sunderland, MA: Sinauer.

Lyons, L. A., Laughlin, T. F., Copeland, N. G., Jenkins, N. A., Womack, J. E., \& O'brien, S. J. (1997). Comparative anchor tagged sequences (CATS) for 
integrative mapping of mammalian genomes. Nature Genetics, 15(1), 47-56. doi:10.1038/ng0197-47

Mackay, T. F., Stone, E. A., \& Ayroles, J. F. (2009). The genetics of quantitative traits: Challenges and prospects. Nature Reviews Genetics, 10(8), 565-577. doi:10.1038/nrg2612

Mafli, A., Wakamatsu, K., \& Roulin, A. (2011). Melanin-based coloration predicts aggressiveness and boldness in captive eastern Hermann's tortoises. Animal Behaviour, 81(4), 859-863. doi:10.1016/j.anbehav.2011.01.025

Meffert, L., Hicks, S., \& Regan, J. (2002). Nonadditive Genetic Effects in Animal Behavior. The American Naturalist, 160(S6). doi:10.1086/342896

Moore, D. S. (2015). The developing genome: An introduction to behavioral epigenetics. Oxford: Oxford University Press.

Mueller, J. C., Korsten, P., Hermannstaedter, C., Feulner, T., Dingemanse, N. J., Matthysen, E., ... Kempenaers, B. (2013). Haplotype structure, adaptive history and associations with exploratory behaviour of the DRD4 gene region in four great tit ( Parus major ) populations. Molecular Ecology, 22(10), 27972809. doi:10.1111/mec.12282

Munafò, M. R., Freimer, N. B., Ng, W., Ophoff, R., Veijola, J., Miettunen, J., . . Flint, J. (2009). 5-HTTLPR genotype and anxiety-related personality traits: A metaanalysis and new data. American Journal of Medical Genetics Part B: Neuropsychiatric Genetics, 150B(2), 271-281. doi:10.1002/ajmg.b.30808 Munafò, M. R., Yalcin, B., Willis-Owen, S. A., \& Flint, J. (2008). Association of the Dopamine D4 Receptor (DRD4) Gene and Approach-Related Personality 
Traits: Meta-Analysis and New Data. Biological Psychiatry, 63(2), 197-206. doi:10.1016/j.biopsych.2007.04.006

Murphy, S. E., Norbury, R., Godlewska, B. R., Cowen, P. J., Mannie, Z. M., Harmer, C. J., \& Munafò, M. R. (2013). The effect of the serotonin transporter polymorphism (5-HTTLPR) on amygdala function: A meta-analysis. Molecular Psychiatry, 18(4), 512-520. doi:10.1038/mp.2012.19

Nelson, R. J., \& Trainor, B. C. (2007). Neural mechanisms of aggression. Nature Reviews Neuroscience, 8(7), 536-546. doi:10.1038/nrn2174

van Oers, K., Drent, P. J., Jong, G. D., \& Noordwijk, A. J. (2004). Additive and nonadditive genetic variation in avian personality traits. Heredity, 93(5), 496503. doi:10.1038/sj.hdy. 6800530

van Oers, K., \& Mueller, J. C. (2010). Evolutionary genomics of animal personality. Philosophical Transactions of the Royal Society B: Biological Sciences, 365(1560), 3991-4000. doi:10.1098/rstb.2010.0178

van Oers, K., Santure, A. W., Cauwer, I. D., Bers, N. E., Crooijmans, R. P., Sheldon, B. C., ... Groenen, M. A. (2013). Replicated high-density genetic maps of two great tit populations reveal fine-scale genomic departures from sex-equal recombination rates. Heredity, 112(3), 307-316. doi:10.1038/hdy.2013.107 van Oers, K., Jong, G. D., Noordwijk, A. V., K., \& Drent, P. (2005). Contribution of genetics to the study of animal personalities: A review of case studies. Behaviour, 142(9), 1185-1206. doi:10.1163/156853905774539364 van Oers, K., Sinn, D.L. Quantitative and Molecular Genetics of Animal Personality. Animal Personalities Behavior, Physiology, and Evolution, 148-200. 
van Oers, K., \& Sinn, D. L. (2011). Toward a Basis for the Phenotypic Gambit: Advances in the Evolutionary Genetics of Animal Personality. From Genes to Animal Behavior Primatology Monographs, 165-183. doi:10.1007/978-4-43153892-9_7

van Oers, K., \& Sinn, D. L. (2011). Toward a Basis for the Phenotypic Gambit: Advances in the Evolutionary Genetics of Animal Personality. From Genes to Animal Behavior Primatology Monographs, 165-183. doi:10.1007/978-4-43153892-9_7

Oortmerssen, G. A., \& Bakker, T. C. (1981). Artificial selection for short and long attack latencies in wildMus musculus domesticus. Behavior Genetics, 11(2), 115-126. doi:10.1007/bf01065622

Ozaki, N., Goldman, D., Kaye, W. H., Plotnicov, K., Greenberg, B. D., Lappalainen, J., . . Murphy, D. L. (2003). Serotonin transporter missense mutation associated with a complex neuropsychiatric phenotype. Molecular Psychiatry, 8(11), 933-936. doi:10.1038/sj.mp.4001365

Page, R. E., Rueppell, O., \& Amdam, G. V. (2012). Genetics of Reproduction and Regulation of Honeybee (Apis melliferaL.) Social Behavior. Annual Review of Genetics, 46(1), 97-119. doi:10.1146/annurev-genet-110711-155610

Penke, L., Denissen, J. J., \& Miller, G. F. (2007). The evolutionary genetics of personality. European Journal of Personality, 21(5), 549-587. doi:10.1002/per.629

Petelle, M. B., Martin, J. G., \& Blumstein, D. T. (2015). Heritability and genetic correlations of personality traits in a wild population of yellow-bellied marmots ( 
Marmota flaviventris ). Journal of Evolutionary Biology, 28(10), 1840-1848. doi:10.1111/jeb.12700

Poissant, J., Réale, D., Martin, J., Festa-Bianchet, M., \& Coltman, D. (2013). A quantitative trait locus analysis of personality in wild bighorn sheep. Ecology and Evolution, 3(3), 474-481. doi:10.1002/ece3.468

Ponsuksili, S., Zebunke, M., Murani, E., Trakooljul, N., Krieter, J., Puppe, B., . . . Wimmers, K. (2015). Integrated Genome-wide association and hypothalamus eQTL studies indicate a link between the circadian rhythm-related gene PER1 and coping behavior. Scientific Reports, 5, 16264. doi:10.1038/srep16264

Quinn, J. L., Patrick, S. C., Bouwhuis, S., Wilkin, T. A., \& Sheldon, B. C. (2009). Heterogeneous selection on a heritable temperament trait in a variable environment. Journal of Animal Ecology, 78(6), 1203-1215. doi:10.1111/j.13652656.2009.01585.x

Quinn, J., \& Cresswell, W. (2005). Personality, anti-predation behaviour and behavioural plasticity in the chaffinch Fringilla coelebs. Behaviour, 142(9), 1377-1402. doi:10.1163/156853905774539391

Rasmuson, M. (2009). Old and new ideas about genes and behaviour. Hereditas, 146(5), 198-203. doi:10.1111/j.1601-5223.2009.02138.x

Ripke, S., O’Dushlaine, C., Chambert, K., Moran, J. L., Kahler, A. K., Akterin, S., ... Sullivan, P. F. (2013). Genome-wide association analysis identifies 13 new risk loci for schizophrenia. Nature Genetics, 45(10), 1150-1159. doi:10.1038/ng.2742

Risch, N. J. (2000). Searching for genetic determinants in the new millennium Nature, 405(6788), 847-856. doi:10.1038/35015718 
Riyahi, S., Björklund, M., Mateos-Gonzalez, F., \& Senar, J. C. (2016). Personality and urbanization: Behavioural traits and DRD4 SNP830 polymorphisms in great tits in Barcelona city. Journal of Ethology. doi:10.1007/s10164-016-04962

Robinson, M. R., Santure, A. W., Decauwer, I., Sheldon, B. C., \& Slate, J. (2013). Partitioning of genetic variation across the genome using multimarker methods in a wild bird population. Molecular Ecology, 22(15), 3963-3980. doi:10.1111/mec. 12375

Rodenburg, T. B., Komen, H., Ellen, E. D., Uitdehaag, K. A., \& Arendonk, J. A. (2008). Selection method and early-life history affect behavioural development, feather pecking and cannibalism in laying hens: A review. Applied Animal Behaviour Science, 110(3-4), 217-228. doi:10.1016/j.applanim.2007.09.009

Roff, D. A. (1996). The Evolution of Genetic Correlations: An Analysis of Patterns. Evolution, 50(4), 1392. doi:10.2307/2410877

Santure, A. W., Poissant, J., Cauwer, I. D., van Oers, K., Robinson, M. R., Quinn, J. L., ... Slate, J. (2015). Replicated analysis of the genetic architecture of quantitative traits in two wild great tit populations. Molecular Ecology, 24(24), 6148-6162. doi:10.1111/mec. 13452

Savitz, J. B., \& Ramesar, R. S. (2004). Genetic variants implicated in personality: A review of the more promising candidates. American Journal of Medical Genetics, 131B(1), 20-32. doi:10.1002/ajmg.b.20155

Scott, A. L., Bortolato, M., Chen, K., \& Shih, J. C. (2008). Novel monoamine oxidase A knock out mice with human-like spontaneous mutation. NeuroReport, 19(7), 739-743. doi:10.1097/wnr.0b013e3282fd6e88 
Shih, J., \& Thompson, R. (1999). Monoamine Oxidase in Neuropsychiatry and Behavior. The American Journal of Human Genetics, 65(3), 593-598. doi:10.1086/302562

Shikano, T., Ramadevi, J., Shimada, Y., \& Merilä, J. (2010). Utility of sequenced genomes for microsatellite marker development in non-model organisms: A case study of functionally important genes in nine-spined sticklebacks (Pungitius pungitius). BMC Genomics, 11(1), 334. doi:10.1186/1471-2164-11334

Shimada, M. K., Inoue-Murayama, M., Ueda, Y., Maejima, M., Murayama, Y., Takenaka, O., . . Ito, S. (2004). Polymorphism in the second intron of dopamine receptor D4 gene in humans and apes. Biochemical and Biophysical Research Communications, 316(4), 1186-1190. doi:10.1016/j.bbrc.2004.03.006

Sih, A., Bell, A., \& Johnson, J. (2004). Behavioral syndromes: An ecological and evolutionary overview. Trends in Ecology \& Evolution, 19(7), 372-378. doi:10.1016/j.tree.2004.04.009

Singh, P., Schimenti, J. C., \& Bolcun-Filas, E. (2014). A Mouse Geneticist's Practical Guide to CRISPR Applications. Genetics, 199(1), 1-15. doi:10.1534/genetics.114.169771

Slate, J. (2013). From Beavis To Beak Color: A Simulation Study To Examine How Much Qtl Mapping Can Reveal About The Genetic Architecture Of Quantitative Traits. Evolution. doi:10.1111/evo.12060 
Slate, J. (2004). INVITED REVIEW: Quantitative trait locus mapping in natural populations: Progress, caveats and future directions. Molecular Ecology, 14(2), 363-379. doi:10.1111/j.1365-294x.2004.02378.x

Sokolowski, M. B. (2001). Drosophila: genetics meets behaviour. Nature Reviews Genetics, 2(11), 879-890. doi:10.1038/35098592

Stearns, F. W. (2010). One Hundred Years of Pleiotropy: A Retrospective. Genetics, 186(3), 767-773. doi:10.1534/genetics.110.122549

Stirling, D. G., Réale, D., \& Roff, D. A. (2002). Selection, structure and the heritability of behaviour. Journal of Evolutionary Biology, 15(2), 277-289. doi:10.1046/j.1420-9101.2002.00389.x

Swiech, L., Heidenreich, M., Banerjee, A., Habib, N., Li, Y., Trombetta, J., . . Zhang, F. (2014). In vivo interrogation of gene function in the mammalian brain using CRISPR-Cas9. Nature Biotechnology, 33(1), 102-106. doi:10.1038/nbt.3055

Taylor, R. W., Boon, A. K., Dantzer, B., Réale, D., Humphries, M. M., Boutin, S., . . Mcadam, A. G. (2012). Low heritabilities, but genetic and maternal correlations between red squirrel behaviours. Journal of Evolutionary Biology, 25(4), 614624. doi:10.1111/j.1420-9101.2012.02456.x

Terracciano, A., Sanna, S., Uda, M., Deiana, B., Usala, G., Busonero, F., . . Costa, P. T. (2008). Genome-wide association scan for five major dimensions of personality. Molecular Psychiatry, 15(6), 647-656. doi:10.1038/mp.2008.113

Tonteri, A., Vasemägi, A., Lumme, J., \& Primmer, C. R. (2010). Beyond MHC: Signals of elevated selection pressure on Atlantic salmon ( Salmo salar ) immune-relevant loci. Molecular Ecology, 19(7), 1273-1282. doi:10.1111/j.1365-294x.2010.04573.x 
Trut, L.N., lliushina, I.Z., Prasolova, L.A., \& Kim, A.A. (1997). The hooded allele and selection of wild Norway rats Rattus norvegicus for behavior. Genetika, 33 (8), 1156-1161. [Translation in English available in: Russian Journal of Genetics, 1997. 33, 983-989]

Trut, L. (1999). Early Canid Domestication: The Farm-Fox Experiment. American Scientist, 87(2), 160. doi:10.1511/1999.20.813

Verhulst, E. C., Mateman, A. C., Zwier, M. V., Caro, S. P., Verhoeven, K. J., \& van Oers, K. (2016). Evidence from pyrosequencing indicates that natural variation in animal personality is associated with DRD4 DNA methylation. Molecular Ecology, 25(8), 1801-1811. doi:10.1111/mec.13519

Visser, M. E., Caro, S. P., van Oers, K.., Schaper, S. V., \& Helm, B. (2010). Phenology, seasonal timing and circannual rhythms: Towards a unified framework. Philosophical Transactions of the Royal Society B: Biological Sciences, 365(1555), 3113-3127. doi:10.1098/rstb.2010.0111

Vitaterna, M. H., Pinto, L. H., \& Takahashi, J. S. (2006). Large-scale mutagenesis and phenotypic screens for the nervous system and behavior in mice. Trends in Neurosciences, 29(4), 233-240. doi:10.1016/j.tins.2006.02.006

Weeland, J., Overbeek, G., Castro, B. O., \& Matthys, W. (2015). Underlying Mechanisms of Gene-Environment Interactions in Externalizing Behavior: A Systematic Review and Search for Theoretical Mechanisms. Clinical Child and Family Psychology Review, 18(4), 413-442. doi:10.1007/s10567-015-0196-4 Wendland, J. R., Martin, B. J., Kruse, M. R., Lesch, K., \& Murphy, D. L. (2006). Simultaneous genotyping of four functional loci of human SLC6A4, with a 
reappraisal of 5-HTTLPR and rs25531. Molecular Psychiatry, 11(3), 224-226. doi:10.1038/sj.mp.4001789

Williams, R. J., Pelton, R. B., \& Siegel, F. L. (1962). Individuality As Exhibited By Inbred Animals; Its Implications For Human Behavior. Proceedings of the National Academy of Sciences, 48(8), 1461-1466. doi:10.1073/pnas.48.8.1461

Youngson, N. A., \& Whitelaw, E. (2008). Transgenerational Epigenetic Effects. Annual Review of Genomics and Human Genetics, 9(1), 233-257. doi:10.1146/annurev.genom.9.081307.164445

Zou, W., \& Zeng, Z. (2009). Multiple interval mapping for gene expression QTL analysis. Genetica, 137(2), 125-134. doi:10.1007/s10709-009-9365-z 